Results First, we observed that up-regulation of costimulatory molecules CD86, CD80 and CD40 induced by BCR cross-linking was down-regulated after IVIg incubation and was associated with a massive reduction of tyrosine phosphorylation. Low mobilisation of intracellular Calcium, which is a hallmark of anergy, was also observed in IVIg-treated B cells. Next, we observed that following BCR stimulation, IVIg blocks BCR aggregation within lipid rafts and increases its internalisation. Consequently, BCR stimulation is not achievable and results in a low activation of the PI3K-Akt signalling pathway. Finally, we demonstrated that IVIg down-regulates $\mathrm{NF \kappa B}$ activation and promotes NFAT transcription factors entry in the nucleus. These findings demonstrated that IVIg induces a selective transcriptional programme, allowing nuclear signals to be independently activated, leading to alternative B cell fates.

Conclusions Our data suggest that IVIg could induce B cells to adopt a state that results in a functional silencing, also called anergy. Our findings provide insights into the effectiveness of IVIg in treating pathologies associated with the loss of B cell tolerance. We also describe a new model to explore the complexity of positive versus negative selection in human B cells.

\section{A5.21 LACK OF IL-27R SIGNALING LEADS TO AN INTRINSIC B CELL DEFECT AND PROTECTION AGAINST CIA}

doi:10.1136/annrheumdis-2013-203219.21

1,20dilia BJ Corneth, 1,2Patrick S Asmawidjaja, ${ }^{3}$ Laurens P Kil, ${ }^{1,2}$ Anne-Marie C Mus, 1,2Franka Luk, ${ }^{1,2}$ Sandra MJ Paulissen, ${ }^{3}$ Rudi W Hendriks, ${ }^{1,2}$ Erik Lubberts. 'Departments of Rheumatology; ${ }^{2}$ Immunology; ${ }^{3}$ Pulmonary Medicine, Erasmus MC, University Medical Center, Rotterdam, The Netherlands

Introduction The IL-27 receptor (IL-27R) is expressed on naïve $\mathrm{T}$ helper cells. Signaling induces Th1 differentiation and IL-10 production and inhibits Th17 differentiation. In addition, the IL-27R is also expressed on B cells, where it is linked to B cell proliferation and IgG2a class switch recombination. However, its role is not fully understood. We therefore aim to determine the role of IL-27R signalling on B cells, both in $\mathrm{T}$ cell independent and in $\mathrm{T}$ cell dependent immune responses.

Methods To determine the effect of IL-27 on B cells, we isolated naïve $B$ cells from wild type mice and cultured them in the absence or presence of rIL-27. T cell dependent responses were investigated using the collagen induced arthritis (CIA) model in wild type and IL-27R deficient (WSX-1 deficient) mice. T cell independent B cell responses were investigated using the TNP-Ficoll immunisation model.

Results Naïve wild type B cells cultured in the presence of rIL-27 showed increased proliferation and expression of activation markers. Next, we investigated the role of IL-27R signalling in vivo in CIA. Both the incidence and severity of CIA were significantly lower in IL-27R deficient mice. Splenic germinal centre B cells were decreased in these mice. In addition, both auto-reactive IgM and IgG2a antibody levels were significantly decreased. These data suggest an impairment of B cell immunity in IL-27R deficient mice. However, CIA also depends on $\mathrm{T}$ cells. To determine whether the impairment of B cell immunity in IL-27R deficient mice was B cell intrinsic, we used the T cell independent TNP-Ficoll immunisation model. Wild type and IL-27R deficient mice were immunised with TNP-Ficoll i.p. and sacrificed seven days later. IL-27R deficient mice had fewer splenic IgM and IgG3 plasma cells and lower serum TNP specific IgM and IgG3 antibody levels.

Conclusions Here we show that IL-27R signalling on B cells is essential for proliferation and activation of B cells. Impaired B cell immunity in IL-27R deficient mice is caused by an intrinsic B cell defect, leading to impaired plasma cell formation and antibody production in both $\mathrm{T}$ cell independent as well as $\mathrm{T}$ cell dependent immune responses.

\section{A5.22 MEMBRANE-BOUND AND SOLUBLE BAFF EXPRESSION BY HUMAN RHEUMATOID FIBROBLAST-LIKE SYNOVIOCYTES IN RESPONSE TO TLR STIMULATION}

doi:10.1136/annrheumdis-2013-203219.22

${ }^{1} \mathrm{NW}$ Kam, ${ }^{2} \mathrm{~A}$ Filer, ${ }^{2} \mathrm{C}$ Buckley, ${ }^{1} \mathrm{M}$ Bombardieri, ${ }^{1} \mathrm{C}$ Pitzalis. 'William Harvey Research Institute, Queen Mary University of London, London, UK; 'Division of Immunity and Infection, College of Medical and Dental Sciences, University of Birmingham, UK

Background and Objectives $B$ cell activating factors of TNF family (BAFF) is associated with the survival and maturation of $B$ cells. BAFF is widely expressed in the rheumatoid arthritis (RA) synovium which is characterised by the presence of synovial niches of autoreactive B cells and sustain in situ autoantibody production. Importantly, B cell niches remain functional in the RA-SCID model in the absence of recirculating cells, suggesting that autocrine mechanisms support ongoing B cell activation in the RA synovium. BAFF exerts its functional role both as a membrane bound protein and in soluble form. Here we investigated whether resident stromal cells in the RA synovium, synovial fibroblasts (RASF), are capable of producing either forms of BAFF and thus contribute to local $B$ cell activation.

Methods mRNA BAFF in RASF stimulated with TLR2, TLR3 and TLR4 ligands was assessed by quantitative Taqman PCR RA dermal fibroblasts (RADF) and osteoarthritis SF (OASF) were used as controls. The cytoplasmic, membrane bound and/or soluble forms of BAFF were investigated by 1) Western blot using total and membrane-enriched protein extracts, 2) flow cytometry, 3) ELISA and 4) immunocytochemistry.

Results In vitro stimulation of TLR3, and to a significantly lesser extent TLR4, but not TLR2 on RASF led to strong induction of BAFF mRNA. In response to TLR3, soluble BAFF was time-dependently released in the supernatant of RASF $(\sim 600 \mathrm{pg} / \mathrm{ml})$ and, to a lesser extent, OASF and RADF. RASF constitutively expressed both cytoplasmic and membrane bound BAFF as demonstrated by WB, FACS and immunocytochemistry which was upregulated upon TLR3 stimulation and was significantly increased as compared to RADF.

Conclusions Here we provide conclusive evidence that SF in the RA synovium are a pivotal source of the B cell survival factor BAFF at both mRNA and protein level. In addition to their significant constitutive expression, RASF can further up-regulate cytoplasmic, membrane-bound and soluble BAFF in response to TLR3 stimulation. Overall, our data strongly support a fundamental role for RASF in sustaining functional B cell activation and antibody production in the inflamed RA synovium.

\section{A5.23 MULTIPARAMETER PHOSPHO-FLOW ANALYSIS OF B CELLS FROM PATIENTS WITH RHEUMATOID ARTHRITIS}

doi:10.1136/annrheumdis-2013-203219.23

'Daniel Szili, ' Judit Pozsgay, 'Zsuzsanna Banko, ${ }^{2}$ Tamas Gati, ${ }^{2,3}$ Gyorgy Nagy, ${ }^{2}$ Bernadett Rojkovich, 'Gabriella Sarmay. 'Department of Immunology, Eötvos Lorand University, Budapest; ${ }^{2}$ Buda Hospital of Hospitaller Brothers of St. John, Budapest; ${ }^{3}$ Department of Rheumatology, Semmelweis University, Budapest, Hungary

Background and Objectives Rheumatoid arthritis (RA) is a common, relapsing autoimmune disease, which affects approximately $1 \%$ of the population worldwide. While the specific molecular events that lead to initiation and onset of RA are not known, an uncontrolled activation of the immune system is considered to be a critical component of the disease. B lymphocytes undoubtedly play a critical role in disease aetiology. Antigen binding to B-cell receptor (BCR) triggers $B$ cell activation, although the threshold of activation can be influenced by other receptors, such as TLR9. TLR9 has received substantial attention as a pathogenic co-stimulator of 